\title{
Routine Multiple Duodenal Biopsy during Endoscopy of Dyspeptic Patients Seems Unnecessary for Screening of Celiac Disease
}

\author{
Amir Behforouz, ${ }^{1}$ Abbas Esmaeelzadeh, ${ }^{2}$ Homan Mosanan Mozaffari, ${ }^{2}$ Ali Mokhtarifar, ${ }^{2}$ \\ Elham Faravani, ${ }^{1}$ Sakineh Amoueian, ${ }^{3}$ Alireza Khooei, ${ }^{3}$ Lida Jarahi, ${ }^{4}$ \\ and Ladan Goshayeshi $\mathbb{C}^{2}$ \\ ${ }^{1}$ Radiology Department, Faculty of Medicine, Mashhad University of Medical Sciences, Mashhad, Iran \\ ${ }^{2}$ Gastroenterology Department, Imam Reza Hospital, Mashhad University of Medical Sciences, Mashhad, Iran \\ ${ }^{3}$ Pathology Department, Mashhad University of Medical Sciences, Mashhad, Iran \\ ${ }^{4}$ Department of Community Medicine, Mashhad University of Medical Sciences, Mashhad, Iran
}

Correspondence should be addressed to Ladan Goshayeshi; goshayeshil@mums.ac.ir

Received 8 October 2020; Revised 13 December 2020; Accepted 16 December 2020; Published 22 December 2020

Academic Editor: Chiara Ricci

Copyright ( 2020 Amir Behforouz et al. This is an open access article distributed under the Creative Commons Attribution License, which permits unrestricted use, distribution, and reproduction in any medium, provided the original work is properly cited.

\begin{abstract}
Introduction. Celiac disease (CD) is a chronic and common cause of dyspepsia with a rising prevalence worldwide. This study is aimed at investigating the prevalence of $\mathrm{CD}$ in dyspeptic patients based on serology and biopsy, determining the associated factors, and assessing the necessity of regular duodenal biopsies from normal mucosa in diagnosis of CD among dyspeptic patients. Methods. This cross-sectional study was performed on 530 adult dyspeptic patients who underwent gastroduodenoscopy in Imam Reza hospital, Mashhad, during 2016-2018. Demographic characteristics, clinical data, and laboratory analyses were extracted from hospital records. CD was diagnosed based on intestinal biopsy and serum antitissue transglutaminase (anti-TTG) levels. Mucosal lesions were classified according to the modified Marsh classification. Data were analyzed in SPSS with $P<0.05$ being considered significant. Results. Overall, 163 males (30.8\%) and 367 females (69.2\%) with an average age of $46.38 \pm 15.54$ years were studied. High anti-TTG levels were seen in $36(6.8 \%)$ patients, and duodenal pathologies were seen in $23(4.5 \%)$ patients. Fifteen $(2.8 \%)$ were diagnosed with CD based on both serology and biopsy. Bloating was the most common type of dyspepsia in CD patients $(7,46.7 \%)$, followed by epigastric pain $(6,40 \%)$, and postprandial fullness $(2,13.3 \%)$. Two CD patients (13.3\%) reported a positive family history for CD. Logistic regression model showed that iron deficiency anemia (IDA), anti-TTG level, and Helicobacter pylori infection were predictors of histological changes of CD, whereas IDA was the only independent predictor of $\mathrm{CD}$ in dyspeptic patients $(\mathrm{OR}=17.65,95 \% \mathrm{CI}=1.53-202.52$, and $P=0.021$ ). Conclusion. $\mathrm{CD}$ is prevalent in dyspeptic patients, but routine biopsy from normal-appearing duodenal mucosa is not recommended for all patients. Serological studies, complete history, and careful endoscopic evaluation may provide better costeffective clinical solutions to improve the diagnostic yield of celiac disease in dyspeptic patients.
\end{abstract}

\section{Introduction}

Celiac disease $(\mathrm{CD})$ is known to be caused by an immune reaction to dietary gluten (a storage protein in wheat, barely, and rye), primarily affecting the small intestine. CD is a common cause of chronic malabsorption of fat-soluble vitamins, iron, and potentially B12 and folic acid. CD can present itself with a variety of gastrointestinal and hepatic symptoms, while many patients are asymptomatic $[1,2]$. CD also commonly causes atypical symptoms such as bloating, nausea, and vomiting $[3,4]$, which frequently delay diagnosis $[1,2]$. It is worth nothing that overlooked CD can lead to a number of long-term complications such as metabolic bone diseases, malignancies, and even mortality [5]. CD is reported to affect about $1 \%$ of the general population in western countries $[6$, $7]$. The advent of highly sensitive serological tests in recent 
decades, along with a higher suspicion of the disease, has led to a marked increase in CD diagnosis $[8,9]$. The diagnosis mainly consists of serological screening of antitissue transglutaminase (anti-TTG) level and upper gastrointestinal endoscopy with multiple duodenal biopsies [10].

Dyspepsia is one of the most common gastrointestinal disorders in the general population with a reported prevalence of about $40 \%[2,11,12]$. CD can present with dyspepsia in up to $24.6 \%$ of patients with CD [13]. The prevalence of $\mathrm{CD}$ in dyspeptic patients is reported to be $0.5-2 \%$, which is two to nine times higher than that of the general population [14-18]. On the one hand, there is insufficient evidence to support the decision to perform multiple duodenal biopsies in dyspeptic patients. On the other hand, most dyspeptic patients do not undergo serological screening for CD [2]. Moreover, the approaches of gastroenterologists vary widely in performing duodenal biopsy for screening of CD in dyspeptic patients [19]. Latest American College of Gastroenterology (ACG) guidelines state that obtaining routine biopsies of normal-appearing duodenal mucosa for diagnosis of CD is not required unless flatulence, anemia, or diabetes is present. However, this recommendation needs more evidence [1].

Few studies have investigated the prevalence of CD in Iranian patients with persistent dyspepsia based on both serological and histological findings. This study is aimed at investigating the prevalence of $\mathrm{CD}$ in dyspeptic patients based on both serology and histology, finding out the factors associated with CD in these patients, and assessing the necessity and efficacy of routine biopsies from normal-appearing duodenal mucosa in detecting $\mathrm{CD}$ in dyspeptic patients.

\section{Materials and Methods}

2.1. Settings and Approval. This cross-sectional study was conducted on adults complaining of dyspepsia who underwent gastroduodenoscopy in Imam Reza hospital, Mashhad, Iran, during 2016-2018. The study protocol was approved by the Ethics Committee of Mashhad University of Medical Sciences (approval code: IR.MUMS.FM.REC.1395.235). Informed consent was obtained from all patients before they enter the study.

2.2. Patients. Keeping an alpha of 0.05 and a $d=0.024$, considering the prevalence of celiac disease among dyspeptic patients in Iranian population, the required sample size was calculated to be 530 patients [15]. Patients who referred to our gastroenterology clinics with chief complaint of dyspepsia during 2016-18, underwent gastroduodenoscopy, and provided informed written consent were recruited using nonrandom available sampling method. All patients were registered in our registry database at the Gastroenterology ward of Imam Reza hospital, and their records were kept for and further follow-ups. Patients who did not incline to participate were excluded from the study.

2.3. Data Collection. Demographic data (including sex and age), clinical presentation (symptoms and type of dyspepsia), complete medical history of comorbidities, and familial his- tory of celiac disease were gathered in the checklists for all patients.

All patients underwent gastroduodenoscopy in a single center by expert gastroenterologists under the same standard conditions, and six biopsy samples were taken from the duodenal mucosa in all of them based on 2013 ACG guidelines [1]. Endoscopic features of CD were categorized in four groups of atrophic duodenal mucosa with loss of folds, scalloping, nodular changes, and fissuring [20]. Results of upper gastrointestinal endoscopy also including Helicobacter pylori infection were compiled in the checklists.

A single expert gastrointestinal pathologist, who was blind to the results of gastroduodenoscopy, performed the histopathological study of all samples. Histological diagnosis of CD was made based on the presence of intraepithelial lymphocytes, hyperplasia of crypts, and/or atrophy of the villi. Results of the histopathological study were classified as absence of CD (degree 0) or suggestive of CD (degrees I to III) according to the modified Marsh criteria [16]. The findings of histopathological study were also recorded in the checklists.

Blood samples $(5 \mathrm{ml})$ were collected from all patients. Complete blood count (CBC), serum iron, ferritin, total iron binding capacity (TIBC), 25-OH-vitamin D3, and anti-TTG immunoglobulin A ( $\operatorname{IgA}$ ) were measured according to standardized methods (using a Diametra kit in a single laboratory) [21]. Iron deficiency anemia (IDA) was defined based on the World Health Organization criteria [22]. Vitamin D deficiency was defined as the respective levels below $20 \mathrm{ng} / \mathrm{ml}$, and vitamin D insufficiency was defined as a serum vitamin D level of $21-29 \mathrm{ng} / \mathrm{m}$ based on the Endocrine Society guidelines [23].

2.4. Statistical Analysis. Data were analyzed using SPSS for windows, version 20, IBM Statistics (Chicago, IL, USA). Chi-square test, Fisher's exact test, independent samples $t$ -test, Mann-Whitney $U$ test, one-way ANOVA test, or Kruskal-Wallis test was used to compare variables in different groups. Binary logistic regression analysis was used to assess the associations between $\mathrm{CD}$ and independent variables. Odds ratio (OR) and 95\% confidence interval (CI) were used to present the findings of regression analyses. A $P<0.05$ was considered statistically significant.

\section{Results and Discussion}

3.1. Results. Overall, 530 dyspeptic patients with a mean age of $46.38 \pm 15.54$ years were included, of whom $163(30.8 \%)$ were male and $367(69.2 \%)$ were female. High anti-TTG levels, suggestive of $\mathrm{CD}$, were seen in 36 (6.8\%) patients. Pathological features of the obtained duodenal samples were compliant with grades I-III of modified Marsh criteria, suggestive of $\mathrm{CD}$, in $23(4.5 \%)$ patients. Only 15 patients $(2.8 \%)$ had both the serological and histopathological criteria and were definitively diagnosed as CD.

Table 1 elaborates the findings regarding the comparison of $\mathrm{CD}$ and non-CD patients. As the table implies, the groups had no significant difference regarding the demographic characteristics $(P>0.05)$. However, CD patients were found 
TABLE 1: Comparison of the characteristics of participants with and without celiac disease.

\begin{tabular}{|c|c|c|c|c|}
\hline Variable & & Non-CD $(N=515)$ & $\mathrm{CD}(N=15)$ & $P$ \\
\hline \multicolumn{5}{|l|}{ Demographics } \\
\hline Age (years) & & $46.11 \pm 15.2$ & $43.27 \pm 12.6$ & $0.43^{*}$ \\
\hline \multirow{2}{*}{ Sex } & Male & $159(30.0 \%)$ & $4(26.0 \%)$ & \multirow{2}{*}{$0.48^{* *}$} \\
\hline & Female & $356(67.0 \%)$ & $11(73.0 \%)$ & \\
\hline \multicolumn{5}{|l|}{ Medical history } \\
\hline \multirow{5}{*}{ Dyspeptic complaint } & Bloating & $113(23.3 \%)$ & $7(46.7 \%)$ & \multirow{5}{*}{$0.09^{*}$} \\
\hline & Epigastric pain & $205(42.3 \%)$ & $6(40.0 \%)$ & \\
\hline & Postprandial fullness & $37(7.6 \%)$ & $2(13.3 \%)$ & \\
\hline & Early satiety & $34(7.0 \%)$ & $0(0.0 \%)$ & \\
\hline & Reflux & $96(19.8 \%)$ & $0(0.0 \%)$ & \\
\hline Family history of CD & & $1(0.2 \%)$ & $2(13.3 \%)$ & $<0.01^{*}$ \\
\hline \multicolumn{5}{|l|}{ Laboratory findings } \\
\hline Anti-TTG (U/ml) & & $3.22 \pm 13.98$ & $150.66 \pm 116.97$ & $<0.01^{* *}$ \\
\hline Abnormal anti-TTG level & & $21(4.1 \%)$ & $15(100.0 \%)$ & $<0.01^{*}$ \\
\hline Hemoglobin (mg/dl) & & $13.32 \pm 1.56$ & $12.08 \pm 1.31$ & $<0.01^{*}$ \\
\hline Ferritin (ng/ml) & & $95.20 \pm 61.38$ & $28.83 \pm 20.29$ & $<0.01^{*}$ \\
\hline TIBC $(\mu \mathrm{g} / \mathrm{dl})$ & & $315.77 \pm 63.44$ & $408.93 \pm 48.25$ & $<0.01^{* *}$ \\
\hline Iron deficiency anemia & & $34(6.6 \%)$ & $6(40.0 \%)$ & $<0.01^{*}$ \\
\hline Vitamin D (ng/ml) & & $18.33 \pm 11.78$ & $17.14 \pm 10.54$ & $0.69^{* *}$ \\
\hline Vitamin D deficiency & & $329(63.9 \%)$ & $11(73.3 \%)$ & $0.32^{*}$ \\
\hline \multicolumn{5}{|l|}{ Endoscopic findings } \\
\hline H. pylori infection & & $2(13.3 \%)$ & $13(86.7 \%)$ & $0.57^{*}$ \\
\hline \multirow{4}{*}{ Histopathological findings of the mucosal specimens } & No atrophy & $487(98.4 \%)$ & $0(0.0 \%)$ & \multirow{4}{*}{$<0.01^{*}$} \\
\hline & Marsh I & $5(1.0 \%)$ & $3(20.0 \%)$ & \\
\hline & Marsh II & $3(0.6 \%)$ & $3(20.0 \%)$ & \\
\hline & Marsh III & $0(0.0 \%)$ & $9(60.0 \%)$ & \\
\hline \multirow{7}{*}{ Duodenoscopy findings } & Normal & $362(73.3 \%)$ & $0(0.0 \%)$ & \multirow{7}{*}{$<0.01^{*}$} \\
\hline & Atrophy & $18(3.6 \%)$ & $7(46.7 \%)$ & \\
\hline & Scalloping & $4(0.8 \%)$ & $1(6.7 \%)$ & \\
\hline & Nodularity & $6(1.2 \%)$ & $3(20.0 \%)$ & \\
\hline & Fissuring & $2(0.4 \%)$ & $3(20.0 \%)$ & \\
\hline & Ulcer & $43(8.7 \%)$ & $0(0.0 \%)$ & \\
\hline & Erosion & $59(11.9 \%)$ & $1(6.7 \%)$ & \\
\hline
\end{tabular}

CD: celiac disease; TTG: tissue transglutaminase; TIBC: total iron binding capacity; H: Helicobacter. ${ }^{*}$ Chi-square test. ${ }^{* *}$ Independent samples $t$-test.

to have higher frequency of positive family history for CD in their first-degree relatives, compared with non-CD patients (13.3\% vs. $0.2 \%, P<0.01$ ). Bloating was the most common manifestation of dyspepsia among the CD patients (7, $46.7 \%$ ), while the respective figure in non-CD patients was epigastric pain $(P=0.09)$.

Upper gastrointestinal endoscopy revealed abnormal appearance of duodenal mucosa in all CD patients (15, $100 \%)$, while only $121(26 \%)$ of non-CD participants had abnormal findings in gastroduodenoscopy $(P<0.01)$. According to modified Marsh classification for histopathological changes in the mucosa, among the $15 \mathrm{CD}$ patients, 3 (20\%) were in grade I, another $3(20 \%)$ were in grade II, and $9(60 \%)$ were in grade III. CD patients showed a significantly higher rate of mucosal atrophy in the sample taken from duodenum $(P<0.01)$. There was no significant difference between $C D$ and non-CD patients regarding the presence of $H$. pylori infection in the biopsy specimens (Table 1).

Mean serum anti-TTG levels were significantly higher in $\mathrm{CD}$ patients than the respective figure in non-CD participants $(P<0.01)$. Abnormal serum levels of anti-TTG were found in 21 non-CD patients (4.1\%), while all $15 \mathrm{CD}$ patients $(100 \%)$ had abnormally high anti-TTG levels $(P<0.01)$. There was no significant association between the level of vitamin $\mathrm{D}$ and $\mathrm{CD}(P=0.69)$. Moreover, the frequency of vitamin $\mathrm{D}$ deficiency was not significantly different between $\mathrm{CD}$ and non-CD patients $(P=0.32)$.

Mean serum levels of ferritin and hemoglobin in patients with CD were significantly lower compared with the non-CD group, while the total iron binding capacity was significantly 
higher in the CD patients $(P<0.01)$. Of the 40 cases with IDA, 6 (15\%) had CD. The frequency of iron deficiency anemia was also significantly higher in the CD group, and there was a significant association between IDA and CD $(P=0.01)$.

Multivariate logistic regression analysis showed that IDA, anti-TTG level, and Helicobacter pylori infection were independent predictors of positive histopathological findings of Marsh I-III, which is compliant with CD (Table 2). However, the only independent predictor of definitive $\mathrm{CD}$, based on both positive serology and pathology, was IDA (odds ratio $=17.65,95 \% \mathrm{CI}=1.538-202.523$, and $P=0.02$ ).

\section{Discussion}

Dyspepsia is a common symptom that may be accompanied by a variety of digestive conditions $[2,11,12]$. Many patients with CD can present with dyspepsia [13], but the prevalence of CD in dyspeptic patients is reportedly around 0.5-2\% [1418]. The necessity of multiple duodenal biopsies for detection of $\mathrm{CD}$ in dyspeptic patients remains under controversy, and there is a paucity of evidence in this regard. Current guidelines suggest that routine biopsies of normal-appearing duodenal mucosa for diagnosis of CD should be justified and are indicated in patients with specific conditions [1]. We aimed to investigate the prevalence of $\mathrm{CD}$ in dyspeptic patients based on both serology and histology and find the factors associated with $\mathrm{CD}$ in these patients. We secondarily aimed to assess the necessity and efficacy of routine biopsies from normal-appearing duodenal mucosa in detecting CD in dyspeptic patients.

Prevalence of $\mathrm{CD}$ in patients with a family history of $\mathrm{CD}$ in first-degree relatives was $13.3 \%$, which is higher than that of the general population (1\%) [6, 7]. Moreover, recent ACG guidelines suggest that the incidence of CD is $20 \%$ in siblings and $10 \%$ in other first-degree relatives [1], which is in line with the results of this study. Our study showed bloating as the most common type of dyspepsia in CD patients. Other studies mention abdominal pain as the most common symptom in CD patients [12].

Abnormally high levels of anti-TTG were recorded in $6.8 \%$ of our dyspeptic patients. This finding is near the results of a previous study on western Iranian population, which reported $7 \%$ serological positivity for CD among patients with functional dyspepsia [24]. In a previous systematic review and meta-analysis of 15 studies, the prevalence of positive serology of CD among dyspeptic patients was reported to be $7.9 \%$ [25], which is comparable to our findings. However, both of the mentioned studies considered either endomysial antibody or anti-TTG as the diagnostic criteria for positive serology, while we only evaluated anti-TTG.

Our results showed the prevalence of positive histopathology for CD among dyspeptic patients to be $4.5 \%$. The pooled prevalence of biopsy-proven CD was reported $1 \%$ (ranged between 0.8 and 2\%) in studies that performed duodenal biopsy as the first-line method, as reported by Ford et al. in their meta-analysis [25]. This inconsistency might be due to the difference in ethnicity and characteristics of the patients.
TABLE 2: Multivariate logistic regression model for the prediction of celiac disease.

\begin{tabular}{|c|c|c|c|c|c|}
\hline \multirow{2}{*}{ Predictor } & \multirow{2}{*}{$\begin{array}{c}\text { Coefficient } \\
\text { (B) }\end{array}$} & \multirow{2}{*}{$\begin{array}{l}\text { Odds } \\
\text { ratio }\end{array}$} & \multicolumn{2}{|c|}{$\begin{array}{l}\text { 95\% confidence } \\
\text { interval }\end{array}$} & \multirow{2}{*}{$P$} \\
\hline & & & $\begin{array}{l}\text { Lower } \\
\text { bound }\end{array}$ & $\begin{array}{l}\text { Upper } \\
\text { bound }\end{array}$ & \\
\hline \multicolumn{6}{|c|}{ Based on positive pathology (Marsh I-III) } \\
\hline IDA & 0.763 & 2.14 & 1.062 & 4.334 & 0.03 \\
\hline Anti-TTG & 1.270 & 3.56 & 1.707 & 7.432 & $<0.01$ \\
\hline $\begin{array}{l}\text { H. pylori } \\
\text { infection }\end{array}$ & 1.425 & 4.16 & 2.224 & 7.780 & $<0.01$ \\
\hline \multicolumn{6}{|c|}{ Based on both positive serology and pathology } \\
\hline IDA & 2.871 & 17.65 & 1.538 & 202.523 & 0.02 \\
\hline
\end{tabular}

IDA: iron deficiency anemia; TTG: tissue transglutaminase; H: Helicobacter.

We found the prevalence of definitive $\mathrm{CD}$ diagnosis to be $2.8 \%$ among our dyspeptic patients, which is higher than the reported figure for general population [6,7]. Our findings are also slightly higher than the reported prevalence for $\mathrm{CD}$ among dyspeptic patients [14-18]. Lasa et al. compared 320 dyspeptic patients with 320 healthy individuals in Argentina and found the prevalence of $\mathrm{CD}$ to be $1.25 \%$ in patients with dyspepsia and $0.62 \%$ in healthy controls [26]. The inconsistencies between our results and those mentioned can be attributed to different patient characteristics as well as different diagnostic criteria for detecting dyspepsia and CD.

A number of factors may influence the decision to perform small bowel biopsy such as type of dyspepsia (e.g., bloating or abdominal pain), IDA, and a family history of CD. Using anti-TTG serology, $6.8 \%$ of the patients were diagnosed with CD, while $4.5 \%$ of patients were identified as CD using histopathological findings of duodenal samples (grades I-III of modified Marsh criteria). Finally, $2.8 \%$ of patients, who had both the serological and histopathological criteria, were diagnosed as definitive $\mathrm{CD}$. Therefore, it can be concluded that performing biopsy of normal-appearing duodenal mucosa in dyspeptic patients during upper gastrointestinal endoscopy yields more specificity in comparison with serological tests.

This study shows that it is helpful to investigate CD through duodenal biopsy only in dyspeptic patients with the endoscopic markers of CD. A few studies recommend routine duodenal biopsy in normal-appearing mucosa while others did not $[2,16,18]$ [27]. Nevertheless, Lecleire et al. state that endoscopic markers of villous atrophy are not able to identify a subgroup of patients who would benefit from duodenal biopsy [28]. Contrary to previous statements, Lasa et al. stated that screening in dyspeptic patients cannot be recommended due to comparable prevalence of $\mathrm{CD}$ in healthy individuals and dyspeptic patients [26].

Our research showed IDA, anti-TTG level, and $H$. pylori infection to be predictive factors for histological findings of Marsh I-III, while IDA was the only predictive factor for definitive diagnosis of $\mathrm{CD}$ in dyspeptic patients. H. pylori infection can lead to duodenal intraepithelial lymphocytosis, being reported as a major cause of lymphocytosis 
duodenosis, after CD [29]. Yet, studies have failed to discover any significant relationships between $H$. pylori and $\mathrm{CD}$ risk [30].

Anemia is the most common nongastrointestinal symptom of $\mathrm{CD}$, and screening of $\mathrm{CD}$ has led to an increase in identification of the etiology behind IDA [1, 31]. In our sample of dyspeptic patients, $15 \%$ of anemic patients had CD. In contrast, the prevalence of biopsy-proven CD in IDA cases has been reported to be $3.2 \%$ in a recent systematic review [32]. The prevalence of $C D$ has reportedly increased among the individuals with unexplained IDA, regardless of the presence of gastrointestinal symptoms [33]. It has been also reported that the severity of $\mathrm{CD}$ was higher in patients with anemia, compared with those without anemia. Therefore, timely diagnosis of $\mathrm{CD}$ is of high clinical importance.

Rather than limiting our sample size and performing HLA DQ2/DQ8 analysis, we included a large number of patients suffering from dyspepsia and tested for both serological and histological findings of CD. In cases with high antiTTG levels and normal biopsy, endoscopy was repeated to increase the accuracy of findings. Moreover, in cases with abnormal pathology and normal serology, anti-TTG IgA levels were rechecked. Nevertheless, our study had some limitations. For instance, including healthy control subjects could have led to a better comparison. In addition, we did not perform endomysial antibody test, which could have added to the accuracy of serological diagnosis. Moreover, we could not follow the patients with high anti-TTG levels but normal biopsy findings, due to the scope of our objectives and limited time span of our research design. A prospective study that can follow such patients would add more insight.

\section{Conclusion}

To conclude, the results showed a higher prevalence of $\mathrm{CD}$ in our sample of dyspeptic patients compared to both general population and dyspeptic patients from other ethnic origins. IDA could be recommended as a predictor for CD. Particularly, CD should be considered in patients with abdominal pain, bloating-type dyspepsia, IDA, and those with a positive family history of CD; therefore, serological screening is recommended in these groups. However, since all patients with $\mathrm{CD}$ showed endoscopic markers of CD in duodenoscopy, it seems that routine duodenal biopsy from normal-appearing mucosa is not recommendable. Nonetheless, biopsy of normal-appearing duodenal mucosa may be feasible in patients with abnormal anti-TTG level, IDA, and $H$. pylori infection.

A key finding of this study is the importance of a preoperative serologic (anti-TTG) test, evaluation of serum ferritin, obtaining clinical and family history, and careful endoscopic evaluation. These steps can provide a cost-effective clinical guideline that will help improve the diagnostic yield of celiac disease in dyspeptic patients.

\section{Data Availability}

The data regarding of this work is available upon reasonable request from the authors. Requests for data will be consid- ered by the corresponding author, who can be contacted at GoshayeshiL@mums.ac.ir.

\section{Conflicts of Interest}

The authors have no conflicts of interest to disclose.

\section{Acknowledgments}

This work was supported by a grant from the Vice Chancellor for Research, Mashhad University of Medical Sciences (grant number 941485).

\section{References}

[1] A. Rubio-Tapia, I. D. Hill, C. P. Kelly, A. H. Calderwood, and J. A. Murray, "ACG clinical guidelines: diagnosis and management of celiac disease," The American Journal of Gastroenterology., vol. 108, no. 5, pp. 656-676, 2013.

[2] L. Petrarca, R. Nenna, G. Mastrogiorgio, M. Florio, M. Brighi, and S. Pontone, "Dyspepsia and celiac disease: prevalence, diagnostic tools and therapy," World Journal of Methodology., vol. 4, no. 3, pp. 189-196, 2014.

[3] D. Craig, G. Robins, and P. D. Howdle, "Advances in celiac disease," Current Opinion in Gastroenterology, vol. 23, no. 2, pp. 142-148, 2007.

[4] A. Fasano and C. Catassi, "Current approaches to diagnosis and treatment of celiac disease: an evolving spectrum," Gastroenterology, vol. 120, no. 3, pp. 636-651, 2001.

[5] A. Rubio-Tapia, R. A. Kyle, E. L. Kaplan et al., "Increased prevalence and mortality in undiagnosed celiac disease," Gastroenterology, vol. 137, no. 1, pp. 88-93, 2009.

[6] K. Mustalahti, C. Catassi, A. Reunanen et al., "The prevalence of celiac disease in Europe: results of a centralized, international mass screening project," Annals of Medicine, vol. 42, no. 8 , pp. 587-595, 2010.

[7] R. Nenna, C. Tiberti, L. Petrarca et al., "The celiac iceberg: characterization of the disease in primary schoolchildren," Journal of Pediatric Gastroenterology and Nutrition, vol. 56, no. 4, pp. 416-421, 2013.

[8] P. H. Green, "The many faces of celiac disease: clinical presentation of celiac disease in the adult population," Gastroenterology, vol. 128, no. 4, pp. S74-S78, 2005.

[9] P. H. Green, A. T. Fleischauer, G. Bhagat, R. Goyal, B. Jabri, and A. I. Neugut, "Risk of malignancy in patients with celiac disease," The American Journal of Medicine., vol. 115, no. 3, pp. 191-195, 2003.

[10] J. I. Allen, D. Katzka, M. Robert, and G. I. Leontiadis, “American Gastroenterological Association Institute guideline on the role of upper gastrointestinal biopsy to evaluate dyspepsia in the adult patient in the absence of visible mucosal lesions: clinical decision support tool," Gastroenterology, vol. 149, no. 4, p. 1119, 2015.

[11] M. Camilleri, D. Dubois, B. Coulie et al., "Prevalence and socioeconomic impact of upper gastrointestinal disorders in the United States: results of the US Upper Gastrointestinal Study," Clinical gastroenterology and hepatology : the official clinical practice journal of the American Gastroenterological Association., vol. 3, no. 6, pp. 543-552, 2005.

[12] M. Rostami Nejad, R. Dabiri, M. J. Ehsani-Ardakani et al., "Gluten associated dyspepsia; serology and histological 
characteristics," Gastroenterology and Hepatology from Bed to Bench., vol. 5, no. 4, pp. 197-201, 2012.

[13] N. J. Talley and N. Vakil, "Guidelines for the management of dyspepsia," The American Journal of Gastroenterology., vol. 100, no. 10, pp. 2324-2337, 2005.

[14] M. T. Bardella, G. Minoli, D. Ravizza et al., "Increased prevalence of celiac disease in patients with dyspepsia," Archives of Internal Medicine, vol. 160, no. 10, pp. 1489-1491, 2000.

[15] M. Rostami Nejad, H. Mahbobipour, Z. Fazeli et al., "Celiac disease in dyspeptic patients," Koomesh Journal., vol. 12, no. 2, pp. 209-214, 2011.

[16] V. M. Lima, L. Gandolfi, J. A. Pires, and R. Pratesi, "Prevalence of celiac disease in dyspeptic patients," Arquivos de Gastroenterologia, vol. 42, no. 3, pp. 153-156, 2005.

[17] E. Ozaslan, S. Akkorlu, E. Eskioglu, and B. Kayhan, "Prevalence of silent celiac disease in patients with dyspepsia," Digestive Diseases and Sciences, vol. 52, no. 3, pp. 692-697, 2007.

[18] E. Giangreco, C. D'agate, C. Barbera et al., "Prevalence of celiac disease in adult patients with refractory functional dyspepsia: value of routine duodenal biopsy," World Journal of Gastroenterology, vol. 14, no. 45, pp. 6948-6953, 2008.

[19] M. Pitman, D. S. Sanders, P. H. R. Green, and B. Lebwohl, "Rates of duodenal biopsy during upper endoscopy differ widely between providers: implications for diagnosis of celiac disease," Journal of Clinical Gastroenterology, vol. 53, no. 2, pp. e61-e67, 2019.

[20] V. H. Shah, H. Rotterdam, D. P. Kotler, A. Fasano, and P. H. Green, "All that scallops is not celiac disease," Gastrointestinal Endoscopy, vol. 51, no. 6, pp. 717-720, 2000.

[21] M. Rostami Nejad, K. Rostami, M. A. Pourhoseingholi et al., "Atypical presentation is dominant and typical for coeliac disease," Journal of Gastrointestinal and Liver Diseases, vol. 18, no. 3, pp. 285-291, 2009.

[22] Organization WH, Iron Deficiency Anaemia: Assessment, Prevention and Control: A Guide for Programme Managers, World Health Organization, 2001.

[23] M. F. Holick, N. C. Binkley, H. A. Bischoff-Ferrari et al., "Evaluation, treatment, and prevention of vitamin D deficiency: an Endocrine Society clinical practice guideline," The Journal of Clinical Endocrinology and Metabolism., vol. 96, no. 7, pp. 1911-1930, 2011.

[24] A. A. Keshavarz, H. Bashiri, A. Ahmadi, and S. BazarganHejazi, "The prevalence of occult celiac disease among patients with functional dyspepsia: a study from the western region of Iran," Gastroenterology Research and Practice, vol. 2010, Article ID 170702, 4 pages, 2010.

[25] A. C. Ford, E. Ching, and P. Moayyedi, "Meta-analysis: yield of diagnostic tests for coeliac disease in dyspepsia," Alimentary Pharmacology \& Therapeutics, vol. 30, no. 1, pp. 28-36, 2009.

[26] J. Lasa, L. Spallone, S. Gandara, E. Chaar, S. Berman, and D. Zagalsky, "Celiac disease prevalence is not increased in patients with functional dyspepsia," Arquivos de Gastroenterologia, vol. 54, no. 1, pp. 37-40, 2017.

[27] K. Robson, M. Alizart, J. Martin, and R. Nagel, "Coeliac patients are undiagnosed at routine upper endoscopy," PLoS One, vol. 9, no. 3, article e90552, 2014.

[28] S. Lecleire, F. di Fiore, M. Antonietti et al., "Endoscopic markers of villous atrophy are not useful for the detection of celiac disease in patients with dyspeptic symptoms," Endoscopy, vol. 38, no. 7, pp. 696-701, 2006.
[29] S. Santolaria, M. Dominguez, J. Alcedo et al., "Lymphocytic duodenosis: etiological study and clinical presentations," Gastroenterología y Hepatología, vol. 36, no. 9, pp. 565-573, 2013.

[30] M. P. Dore, R. Salis, M. F. Loria, V. Villanacci, G. Bassotti, and G. M. Pes, "Helicobacter pylori infection and occurrence of celiac disease in subjects HLA-DQ2/DQ8 positive: a prospective study," Helicobacter, vol. 23, no. 2, article e12465, 2018.

[31] A. Ganji, A. Esmaielzadeh, M. Aafzal Aghayee, L. Goshayeshi, and K. Ghaffarzadegan, "The clinical presentation of celiac disease: experiences from northeastern Iran," Middle East Journal of Digestive Diseases., vol. 6, no. 2, pp. 93-97, 2014.

[32] S. Mahadev, M. Laszkowska, J. Sundström, M. Björkholm, B. Lebwohl, P. H. Green et al., "Prevalence of Celiac Disease in Patients With Iron Deficiency Anemia-A Systematic Review With Meta-analysis," Gastroenterology, vol. 155, no. 2, pp. 374-382.e1, 2018.

[33] M. F. Kagnoff, "AGA institute medical position statement on the diagnosis and management of celiac disease," Gastroenterology, vol. 131, no. 6, pp. 1977-1980, 2006. 